\title{
ON DIRICHLET'S PRINCIPLE AND PROBLEM
}

\author{
PER ÅHAG, URBAN CEGRELL and RAFAŁ CZYŻ
}

\begin{abstract}
The aim of this paper is to give a new proof of the complete characterization of measures for which there exists a solution of the Dirichlet problem for the complex Monge-Ampère operator in the set of plurisubharmonic functions with finite pluricomplex energy. The proof uses variational methods.
\end{abstract}

\section{Introduction}

Throughout this note let $\Omega \subseteq \mathrm{C}^{n}, n \geq 1$, be a bounded, connected, open, and hyperconvex set. By $\mathscr{E}_{0}$ we denote the family of all bounded plurisubharmonic functions $\varphi$ defined on $\Omega$ such that

$$
\lim _{z \rightarrow \xi} \varphi(z)=0 \quad \text { for every } \quad \xi \in \partial \Omega, \quad \text { and } \int_{\Omega}\left(d d^{c} \varphi\right)^{n}<\infty,
$$

where $\left(d d^{c} \cdot\right)^{n}$ is the complex Monge-Ampère operator. Next let $\mathscr{E}_{p}, p>0$, denote the family of plurisubharmonic functions $u$ defined on $\Omega$ such that there exists a decreasing sequence $\left\{u_{j}\right\}, u_{j} \in \mathscr{E}_{0}$, that converges pointwise to $u$ on $\Omega$, as $j$ tends to $\infty$, and

$$
\sup _{j \geq 1} \int_{\Omega}\left(-u_{j}\right)^{p}\left(d d^{c} u_{j}\right)^{n}=\sup _{j \geq 1} e_{p}\left(u_{j}\right)<\infty .
$$

If $u \in \mathscr{E}_{p}$, then $e_{p}(u)<\infty$ ([10], [14]). It should be noted that it follows from [10] that the complex Monge-Ampère operator is well-defined on $\mathscr{E}_{p}$. It is not only within pluripotential theory these cones have been proven useful, but also as a tool in dynamical systems and algebraic geometry (see, e.g., [2], [17]). For further information on pluripotential theory we refer to [16], [19], [20].

The purpose of this paper is to give a new proof of Theorem B below and use Theorem B to prove that (2) implies (1) in the following theorem:

Received 9 November 2010. 
Theorem A. Let $\mu$ be a non-negative Radon measure. Then the following conditions are equivalent:

(1) there exists a function $u \in \mathscr{E}_{1}$ such that $\left(d d^{c} u\right)^{n}=\mu$,

(2) there exists a constant $B>0$, such that

$$
\int_{\Omega}(-\varphi) d \mu \leq B e_{1}(\varphi)^{\frac{1}{n+1}} \quad \text { for all } \quad \varphi \in \mathscr{E}_{1},
$$

(3) the class $\mathscr{E}_{1}$ is contained in $L^{1}(\mu)$,

(4) the class $\mathscr{E}_{1}$ is contained in $L^{1}(\mu)$. Furthermore, for any sequence $\left\{v_{j}\right\} \subset$ $\mathscr{E}_{1}$ such that $e_{1}\left(v_{j}\right) \leq 1$, there exists a subsequence $\left\{v_{j_{k}}\right\}$ of $\left\{v_{j}\right\}$ that is convergent in the $L^{1}(\mu)$ topology.

In [10], the second-named author proved that the two first conditions in Theorem A are equivalent. This gives a complete characterization of measures for which there exists a solution of the Dirichlet problem for the complex Monge-Ampère operator in $\mathscr{E}_{1}$.

Before we continue we need some more notation. We say that a non-negative Radon measure $\mu$ belongs to $\mathscr{M}_{1}$ if there exists constant $A \geq 0$ such that

$$
\int_{\Omega}(-u) d \mu \leq A e_{1}(u)^{\frac{1}{n+1}}
$$

holds for all $u \in \mathscr{E}_{1}$. For $\mu \in \mathscr{M}_{1}$, let the functional $\mathscr{J}_{\mu}: \mathscr{E}_{1} \rightarrow \mathrm{R}$ be defined by

$$
\mathscr{J}_{\mu}(u)=\frac{1}{n+1} \int_{\Omega}(-u)\left(d d^{c} u\right)^{n}+\int_{\Omega} u d \mu=\frac{1}{n+1} e_{1}(u)-\|u\|_{1} .
$$

Theorem B. Let $\mu \in \mathscr{M}_{1}$, and $u \in \mathscr{E}_{1}$. Then the following assertions are equivalent

(1) $\left(d d^{c} u\right)^{n}=d \mu$,

(2) $\mathscr{J}_{\mu}(u)=\inf _{w \in \mathscr{E}_{1}} \mathscr{J}_{\mu}(w)$.

Theorem B gives a characterization of solutions $u$ of the Dirichlet problem $\left(d d^{c} u\right)^{n}=\mu$ as a minimizing functions for the functional $\mathscr{J}_{\mu}$ defined by the measure $\mu$. This theorem was first proved by Bedford and Taylor for $\mu=0$, in the class of locally bounded plurisubharmonic functions ([6], [7]). Later Kalina proved the Dirichlet principle with some additional assumptions on $\mu$ and $u$ ([18]). Using that the first two conditions in Theorem A are equivalent, Persson [21], proved the Dirichlet principle in $\mathscr{E}_{1}$. In this note we prove Theorem B without using Theorem A. 
In the process of writing this note we have not only been inspired by Bedford's and Taylor's, Kalina's and Persson's pioneer work, but also of the recent work by Berman et al. [9]. The authors would also like to express their gratitude to Robert Berman and Sebastien Boucksom for valuable discussions and comments on an earlier version of this paper.

\section{Preliminaries}

We shall need the following theorem.

TheOREM 2.1. Let $p>0$, and $n \geq 2$. Then there exists a constant $D(n, p) \geq 1$, depending only on $n$ and $p$, such that for any $u_{0}, u_{1}, \ldots, u_{n} \in \mathscr{E}_{p}$ it holds that

$$
\begin{aligned}
\int_{\Omega}\left(-u_{0}\right)^{p} d d^{c} u_{1} & \wedge \cdots \wedge d d^{c} u_{n} \\
& \leq D(n, p) e_{p}\left(u_{0}\right)^{p /(p+n)} e_{p}\left(u_{1}\right)^{1 /(n+p)} \ldots e_{p}\left(u_{n}\right)^{1 /(n+p)} .
\end{aligned}
$$

Furthermore, $D(n, 1)=1$ and $D(n, p)>1$ for $p \neq 1$.

Proof. See Theorem 3.4 in [21] (see also [3], [5], [10], [15]).

For $p \neq 1$ the constant $D(n, p)$, in Theorem 2.1 , is strictly great than 1 ([3], [4]). For this reason we can not use similar variational method to prove the Dirichlet principle in $\mathscr{E}_{p}$ when $p \neq 1$.

Lemma 2.2. For all $u, v \in \mathscr{E}_{1}$ we have that

$$
e_{1}(u+v)^{\frac{1}{n+1}} \leq e_{1}(u)^{\frac{1}{n+1}}+e_{1}(v)^{\frac{1}{n+1}} .
$$

Furthermore, if $\mu \in \mathcal{M}_{1}$, then $\mathscr{J}_{\mu}$ is convex, and if $\left\{u_{j}\right\} \subset \mathscr{E}_{1},\left\|u_{j}\right\|_{1} \rightarrow \infty$, then $\mathscr{J}_{\mu}\left(u_{j}\right) \rightarrow \infty$.

Proof. The first statement, the triangular type inequality for $e_{1}(u)^{\frac{1}{n+1}}$, follows from Theorem 2.1 since

$$
e_{1}(u+v) \leq e_{1}(u)^{\frac{1}{n+1}} e_{1}(u+v)^{\frac{n}{n+1}}+e_{1}(v)^{\frac{1}{n+1}} e_{1}(u+v)^{\frac{n}{n+1}} .
$$

In particular, $e_{1}(u)^{\frac{1}{n+1}}$ is convex, and under the assumption that $\mu \in \mathscr{M}_{1}$ the functional $\mathscr{J}_{\mu}$ is also convex. From the definition of $\mathscr{M}_{1}$ it follows that there exists constant $A>0$ such that

$$
\|u\|_{1} \leq A e_{1}(u)^{\frac{1}{1+n}}, \quad \text { for all } \quad u \in \mathscr{E}_{1} .
$$

If $\left\|u_{j}\right\|_{1} \rightarrow \infty$, then $e_{1}\left(u_{j}\right) \rightarrow \infty$, and therefore we get that

$$
\mathscr{J}_{\mu}\left(u_{j}\right)=\frac{1}{n+1} e_{1}\left(u_{j}\right)-\left\|u_{j}\right\|_{1} \geq \frac{1}{n+1} e_{1}\left(u_{j}\right)-A e_{1}\left(u_{j}\right)^{\frac{1}{n+1}} \rightarrow \infty .
$$


This completes this proof.

Lemma 2.3. Let $v, u \in \mathscr{E}_{1}(\Omega)$, and $w \in \mathscr{E}_{1} \cap C(\Omega)$. Then

$$
\int_{\{w<u\}}(-v)\left(d d^{c} u\right)^{n} \leq \int_{\{w<u\}}(-v)\left(d d^{c} w\right)^{n} .
$$

Proof. Assume first that $v, w \in \mathscr{E}_{1} \cap C(\Omega)$. Without loss of generality we can assume that $\int_{\{u=w\}}(-v)\left(d d^{c} w\right)^{n}=0$. The measure $\left(d d^{c} w\right)^{n}$ vanishes on pluripolar sets, and therefore we have that

$$
\int_{\{u=r w\}}(-v)\left(d d^{c} w\right)^{n}=0,
$$

except for at most denumerably many $r$. Lemma 5.4 in [10] yields that

$$
\begin{aligned}
\int_{\{w<u\}} & (-v)\left(d d^{c} u\right)^{n} \\
= & \int_{\{w<u\}}(-v)\left(d d^{c} \max (u, w)\right)^{n} \\
= & \int_{\Omega}(-v)\left(d d^{c} \max (w, u)\right)^{n}+\int_{\{w \geq u\}} v\left(d d^{c} \max (u, w)\right)^{n} \\
\leq & \int_{\Omega}(-v)\left(d d^{c} w\right)^{n}+\int_{\{w>u\}} v\left(d d^{c} \max (u, w)\right)^{n} \\
= & \int_{\{w<u\}}(-v)\left(d d^{c} w\right)^{n}+\int_{\{u=w\}}(-v)\left(d d^{c} w\right)^{n} .
\end{aligned}
$$

Thus, inequality (2) holds if $v \in \mathscr{E}_{1} \cap C(\Omega)$. An approximation of $v \in \mathscr{E}_{1}(\Omega)$ by a decreasing sequence in $\mathscr{E}_{1} \cap C(\Omega)$ completes the proof (see, e.g., [11], [12]).

Lemma 2.4. Let $u, v \in \mathscr{E}_{1}$, and assume that $v$ is continuous. For $t \in \mathrm{R}$, set

$$
P(u+t v)=\sup \left\{w \in \mathscr{E}_{1}: w \leq u+t v\right\} .
$$

Then $P(u+t v) \in \mathscr{E}_{1}$, and for $s, t \in \mathrm{R}$ we have that

$$
|P(u+t v)-P(u+s v)| \leq|t-s|(-v) .
$$

Proof. If $t \geq 0$, then

$$
P(u+t v)=u+t v,
$$


and in the case $s, t \geq 0$ the lemma is obvious. Furthermore, when $t<0$, the function $P(u+t v)$ is upper semicontinuous, and $u \leq P(u+t v) \leq u+t v$. Thus, $P(u+t v) \in \mathscr{E}_{1}$. For $s<t<0$, we have that

$$
P(u+t v) \leq P(u+s v) \quad \text { and } \quad P(u+s v)+(t-s) v \leq P(u+t v) .
$$

Hence, $|P(u+t v)-P(u+s v)| \leq|t-s|(-v)$. Similarly one can prove that if $t \leq 0 \leq s$, then

$$
-s v \leq P(u+t v)-P(u+s v) \leq(s-t)(-v) .
$$

Lemma 2.5. Let $u, v \in \mathscr{E}_{1}$, and assume that $v$ is continuous. For any $0 \leq k \leq n$, we have that

(3) $\lim _{t \rightarrow 0^{-}} \int_{\Omega} \frac{P(u+t v)-t v-u}{t}\left(d d^{c} u\right)^{k} \wedge\left(d d^{c} P(u+t v)\right)^{n-k}=0$.

In particular,

(4) $\lim _{t \rightarrow 0^{-}} \int_{\Omega} \frac{P(u+t v)-u}{t}\left(d d^{c} u\right)^{k} \wedge\left(d d^{c} P(u+t v)\right)^{n-k}=\int_{\Omega} v\left(d d^{c} u\right)^{n}$.

Proof. For $t<0$, consider the function $h(t)=\frac{P(u+t v)-t v-u}{t}$. A straightforward calculation shows that $h$ is a decreasing function, and

$$
0 \leq \frac{P(u+t v)-t v-u}{t} \leq-v .
$$

Hence, for fixed $s<0$ we have that

$$
\begin{aligned}
\lim _{t \rightarrow 0^{-}} & \int_{\Omega} \frac{P(u+t v)-t v-u}{t}\left(d d^{c} u\right)^{k} \wedge\left(d d^{c} P(u+t v)\right)^{n-k} \\
& \leq \lim _{t \rightarrow 0^{-}} \int_{\Omega} \frac{P(u+s v)-s v-u}{s}\left(d d^{c} u\right)^{k} \wedge\left(d d^{c} P(u+t v)\right)^{n-k} \\
& =\int_{\Omega} \frac{P(u+s v)-s v-u}{s}\left(d d^{c} u\right)^{n} \leq \int_{\{P(u+s v)-s v<u\}}(-v)\left(d d^{c} u\right)^{n} .
\end{aligned}
$$

Let $u_{k} \in \mathscr{E}_{0} \cap C(\Omega)$ be a decreasing sequence that converges to $u$ such that

$$
\int_{\{P(u+s v)-s v<u\}}(-v)\left(d d^{c} u\right)^{n} \leq 2 \int_{\left\{P\left(u_{k}+s v\right)-s v<u\right\}}(-v)\left(d d^{c} u\right)^{n}
$$


(see, e.g., [11], [12]). We can apply Lemma 2.3 to conclude that

$$
\begin{aligned}
& \int_{\left\{P\left(u_{k}+s v\right)-s v<u\right\}}(-v)\left(d d^{c} u\right)^{n} \\
& \quad \leq \int_{\left\{P\left(u_{k}+s v\right)-s v<u_{k}\right\}}(-v)\left(d d^{c}\left(P\left(u_{k}+s v\right)-s v\right)\right)^{n} \\
& \leq-s M \rightarrow 0, \quad \text { as } \quad s \rightarrow 0,
\end{aligned}
$$

where $M$ is a constant only depending on $n,\|v\|$, and $\int_{\Omega} v\left(d d^{c}(u+v)\right)^{n}$. Here we have used that

$$
\int_{\left\{P\left(u_{k}+s v\right)<u_{k}+s v\right\}}\left(d d^{c}\left(P\left(u_{k}+s v\right)\right)\right)^{n}=0 .
$$

This is a consequence of Corollary 9.2 in [8]. The equality (4) is then a consequence of the equality (3), and the proof is complete.

Lemma 2.6. Let $u, v \in \mathscr{E}_{1}$, and assume that $v$ is continuous. For $t \geq 0$, we set

$$
f(t)=\int_{\Omega}-(u+t v)\left(d d^{c}(u+t v)\right)^{n}=e_{1}(u+t v) .
$$

Then

$$
f^{\prime}\left(0^{+}\right)=(n+1) \int_{\Omega}(-v)\left(d d^{c} u\right)^{n}
$$

where

$$
f^{\prime}\left(0^{+}\right)=\lim _{t \rightarrow 0^{+}} \frac{f(t)-f(0)}{t} .
$$

Proof. This is an immediate consequence of the construction.

Lemma 2.7. Let $u, v \in \mathscr{E}_{1}$, and assume that $v$ is continuous. For $t<0$, we set

$$
f(t)=\int_{\Omega}(-P(u+t v))\left(d d^{c} P(u+t v)\right)^{n}=e_{1}(P(u+t v)) .
$$

Then

$$
f^{\prime}\left(0^{-}\right)=(n+1) \int_{\Omega}(-v)\left(d d^{c} u\right)^{n}
$$

where

$$
f^{\prime}\left(0^{-}\right)=\lim _{t \rightarrow 0^{-}} \frac{f(t)-f(0)}{t} .
$$


Proof. Note that

$$
\begin{aligned}
\frac{1}{t}\left(\int_{\Omega}(-P(u+\right. & \left.t v))\left(d d^{c} P(u+t v)\right)^{n}-\int_{\Omega}(-u)\left(d d^{c} u\right)^{n}\right) \\
& =\sum_{k=0}^{n} \int_{\Omega} \frac{u-P(u+t v)}{t}\left(d d^{c} u\right)^{k} \wedge\left(d d^{c} P(u+t v)\right)^{n-k},
\end{aligned}
$$

and then Lemma 2.5 completes the proof.

Corollary 2.8. Let $u, v \in \mathscr{E}_{1}$, and assume that $v$ is continuous. Then it holds that

$$
\begin{aligned}
& \mathscr{J}_{\mu}(P(u+t v))^{\prime}\left(0^{-}\right) \\
& \quad=\left(\frac{1}{n+1} \int_{\Omega}(-P(u+t v))\left(d d^{c} P(u+t v)\right)^{n}+\int_{\Omega} P(u+t v) d \mu\right)^{\prime}\left(0^{-}\right) \\
& \quad \geq \int_{\Omega}(-v)\left(d d^{c} u\right)^{n}+\int_{\Omega} v d \mu .
\end{aligned}
$$

Proof. The existence of $\mathscr{J}_{\mu}(P(u+t v))^{\prime}\left(0^{-}\right)$follows from Lemma 2.7 and the fact that the function $t \rightarrow \frac{P(u+t v)-u}{t}$ is decreasing. For $t<0$

$$
\begin{aligned}
\int_{\Omega} \frac{(P(u+t v)-u)}{t} d \mu & =\int_{\Omega} \frac{(P(u+t v)-t v-u)}{t} d \mu+\int_{\Omega} v d \mu \\
& \geq \int_{\Omega} v d \mu
\end{aligned}
$$

and the proof is finished by Lemma 2.7 .

\section{Proof of Theorem B}

Proof. Let $\mu \in \mathcal{M}_{1}$, and $u \in \mathscr{E}_{1}$.

$(1) \Rightarrow(2)$ : Assume that $\left(d d^{c} u\right)^{n}=d \mu$, and let $v \in \mathscr{E}$. Then by Theorem 2.1, and Young's inequality we get that

$$
\begin{aligned}
\int_{\Omega}(-v) d \mu & =\int_{\Omega}(-v)\left(d d^{c} u\right)^{n} \leq e_{1}(v)^{\frac{1}{n+1}} e_{1}(u)^{\frac{n}{n+1}} \\
& \leq \frac{1}{n+1} e_{1}(v)+\frac{n}{n+1} e_{1}(u) .
\end{aligned}
$$

Hence

$$
\mathscr{J}_{\mu}(v)=\frac{1}{n+1} e_{1}(v)+\int_{\Omega} v d \mu \geq-\frac{n}{n+1} e_{1}(u)=\mathscr{J}_{\mu}(u) .
$$


Thus, $\mathscr{J}_{\mu}(u)=\inf _{w \in \mathscr{E}_{1}} \mathscr{J}_{\mu}(w)$.

(2) $\Rightarrow(1)$ : Let $u \in \mathscr{E}_{1}$ be such that $\mathscr{J}_{\mu}(u)=\inf _{w \in \mathscr{E}_{1}} \mathscr{J}_{\mu}(w)$. Take an arbitrary function $v$ in $\mathscr{E}_{1} \cap C(\Omega)$, and define

$$
g(t)=\mathscr{J}_{\mu}(P(u+t v)) .
$$

This construction yields that that $0 \geq g^{\prime}\left(0^{-}\right)$, and $g^{\prime}\left(0^{+}\right) \geq 0$, Since for all $t$ we have that $g(0) \leq g(t)$. The existence of $g^{\prime}\left(0^{+}\right)$and $g^{\prime}\left(0^{-}\right)$follows from Lemma 2.6 and Corollary 2.8 respectively. The last inequality and Lemma 2.6 gives us that

$$
\int_{\Omega}(-v)\left(d d^{c} u\right)^{n}+\int_{\Omega} v d \mu \geq 0,
$$

and therefore it follows from Corollary 2.8 that $g^{\prime}\left(0^{-}\right)=0$, and

$$
0=\int_{\Omega}(-v)\left(d d^{c} u\right)^{n}+\int_{\Omega} v d \mu .
$$

Thus,

$$
\int_{\Omega} v d \mu=\int_{\Omega} v\left(d d^{c} u\right)^{n} .
$$

Lemma 3.1 in [11] yields that $\left(d d^{c} u\right)^{n}=d \mu$, since $v$ was an arbitrary function in $\mathscr{E}_{1} \cap C(\Omega)$.

REMARK 1. The uniqueness of the solution for the equation $\left(d d^{c} u\right)^{n}=d \mu$ follows from the comparison principle (see, e.g., [1], [10]). Using Lemma 2.2, uniqueness in $\mathscr{E}_{1}$ can be obtained in the following way.

Proposition 3.1. For any $\mu \in \mathscr{M}_{1}$ there exists at most one function $u \in \mathscr{E}_{1}$ for which the functional $\mathscr{J}_{\mu}$ achieves its infimum on $\mathscr{E}_{1}$. In other words, there exists at most one solution $u \in \mathscr{E}_{1}$ for the complex Monge-Ampère equation $\left(d d^{c} u\right)^{n}=\mu$.

Proof. Let $S$ denote the set of solutions to Dirichlet's problem with given measure $\mu \in \mathscr{M}_{1}$. Then we know by Lemma 2.2 that $\mathrm{S}$ is a convex set. Assume that there exist functions $u, v \in S$. Then also $t u+(1-t) v \in S$, for $0 \leq t \leq 1$. Furthermore, we have that for any $1 \leq k \leq n$, and all $\varphi \in \mathscr{E}_{0}$, it holds that

$$
\begin{aligned}
\int_{\Omega}(-\varphi)\left(d d^{c} u\right)^{k} \wedge\left(d d^{c} v\right)^{n-k} & \leq\left(\int_{\Omega}(-\varphi)\left(d d^{c} u\right)^{n}\right)^{\frac{k}{n}}\left(\int_{\Omega}(-\varphi)\left(d d^{c} v\right)^{n}\right)^{\frac{n-k}{n}} \\
& \leq \int_{\Omega}(-\varphi) d \mu .
\end{aligned}
$$


This implies that for all $1 \leq k \leq n$ we have that

$$
(-\varphi)\left(d d^{c} u\right)^{k} \wedge\left(d d^{c} v\right)^{n-k}=(-\varphi) d \mu,
$$

since otherwise we would have that $t u+(1-t) v \notin S$. From (5) it follows that

$$
\left(d d^{c} u\right)^{k} \wedge\left(d d^{c} v\right)^{n-k}=d \mu .
$$

Now we can use an argument from the proof of Theorem 3.15 in [13] to prove that $u=v$. By [12], there exists a strictly plurisubharmonic exhaustion function $\psi \in \mathscr{E}_{0} \cap C^{\infty}(\Omega)$ for $\Omega$. To complete the proof it is enough to show that

$$
\int_{\Omega} d(u-v) \wedge d^{c}(u-v) \wedge\left(d d^{c} \psi\right)^{n-1}=0 .
$$

We shall prove this statement by induction. It is easy to see that

$$
0=\int_{\Omega} d(u-v) \wedge d^{c}(u-v) \wedge\left(d d^{c} u\right)^{k} \wedge\left(d d^{c} v\right)^{l} \wedge d d^{c} \psi,
$$

for $k+l=n-2$.

Assume that

$$
0=\int_{\Omega} d(u-v) \wedge d^{c}(u-v) \wedge\left(d d^{c} u\right)^{k} \wedge\left(d d^{c} v\right)^{l} \wedge\left(d d^{c} \psi\right)^{p},
$$

for $k+l=n-1-p$.

Then, for $k+l=n-2-p$ we have that

$$
\begin{aligned}
0 \leq & \int_{\Omega} d(u-v) \wedge d^{c}(u-v) \wedge\left(d d^{c} u\right)^{k} \wedge\left(d d^{c} v\right)^{l} \wedge\left(d d^{c} \psi\right)^{p+1} \\
= & \int_{\Omega}-(u-v) d d^{c}(u-v) \wedge\left(d d^{c} u\right)^{k} \wedge\left(d d^{c} v\right)^{l} \wedge\left(d d^{c} \psi\right)^{p+1} \\
= & \int_{\Omega}-\psi\left(d d^{c}(u-v)\right)^{2} \wedge\left(d d^{c} u\right)^{k} \wedge\left(d d^{c} v\right)^{l} \wedge\left(d d^{c} \psi\right)^{p} \\
= & \int_{\Omega} d \psi \wedge d^{c}(u-v) \wedge d d^{c}(u-v) \wedge\left(d d^{c} u\right)^{k} \wedge\left(d d^{c} v\right)^{l} \wedge\left(d d^{c} \psi\right)^{p} \\
\leq & \left|\int_{\Omega} d \psi \wedge d^{c}(u-v) \wedge d d^{c} u \wedge\left(d d^{c} u\right)^{k} \wedge\left(d d^{c} v\right)^{l} \wedge\left(d d^{c} \psi\right)^{p}\right| \\
& +\left|\int_{\Omega} d \psi \wedge d^{c}(u-v) \wedge d d^{c} v \wedge\left(d d^{c} u\right)^{k} \wedge\left(d d^{c} v\right)^{l} \wedge\left(d d^{c} \psi\right)^{p}\right|
\end{aligned}
$$




$$
\begin{aligned}
\leq & \left(\int_{\Omega} d \psi \wedge d^{c} \psi \wedge\left(d d^{c} u\right)^{k+1} \wedge\left(d d^{c} v\right)^{l} \wedge\left(d d^{c} \psi\right)^{p}\right. \\
& \left.\times \int_{\Omega} d(u-v) \wedge d^{c}(u-v) \wedge\left(d d^{c} u\right)^{k+1} \wedge\left(d d^{c} v\right)^{l} \wedge\left(d d^{c} \psi\right)^{p}\right)^{\frac{1}{2}} \\
& +\left(\int_{\Omega} d \psi \wedge d^{c} \psi \wedge\left(d d^{c} u\right)^{k} \wedge\left(d d^{c} v\right)^{l+1} \wedge\left(d d^{c} \psi\right)^{p}\right. \\
& \left.\times \int_{\Omega} d(u-v) \wedge d^{c}(u-v) \wedge\left(d d^{c} u\right)^{k} \wedge\left(d d^{c} v\right)^{l+1} \wedge\left(d d^{c} \psi\right)^{p}\right)^{\frac{1}{2}} \\
= & 0 .
\end{aligned}
$$

\section{Proof of Theorem A}

We shall need the following lemma.

Lemma 4.1. Let $\mu$ be a non-negative Radon measure such that $\mu(\Omega)<\infty$. If there exists a constant $A>0$ such that

$$
\int_{\Omega}(-\varphi)^{2} d \mu \leq A e_{1}(\varphi)^{\frac{2}{n+1}} \quad \text { for all } \varphi \in \mathscr{E}_{1},
$$

then $\mu \in \mathcal{M}_{1}$. Furthermore, for any sequence $\left\{v_{j}\right\} \subset \mathscr{E}_{1}$ such that $e_{1}\left(v_{j}\right) \leq 1$, there exists a subsequence $\left\{v_{j_{k}}\right\}$ of $\left\{v_{j}\right\}$ that converges to a plurisubharmonic function in the $L^{1}(\mu)$ topology. Finally, there exists a uniquely determined function $u \in \mathscr{E}_{1}$, with $\left(d d^{c} u\right)^{n}=\mu$.

Proof. Assume that $\mu$ is a non-negative Radon measure with $\mu(\Omega)<\infty$, and take a function $\varphi \in \mathscr{E}_{1}$. Then it follows from inequality (6) that there exists a constant $A>0$ such that

$$
\begin{aligned}
\int_{\Omega}(-\varphi) d \mu \leq\left(\int_{\Omega}(-\varphi)^{2} d \mu\right)^{1 / 2} \mu(\Omega)^{1 / 2} & \leq A^{1 / 2} e_{1}(\varphi)^{\frac{1}{n+1}} \mu(\Omega)^{1 / 2} \\
& =C e_{1}(\varphi)^{\frac{1}{n+1}}<\infty
\end{aligned}
$$

where $C=A^{1 / 2} \mu(\Omega)^{1 / 2}$. Thus, $\mathscr{E}_{1} \subseteq L^{1}(\mu)$.

Assume now that $\left\{v_{j}\right\} \subset \mathscr{E}_{1}$ is a sequence such that

$$
\sup _{j} e_{1}\left(v_{j}\right) \leq 1
$$

We can then pick a subsequence, again denoted by $\left\{v_{j}\right\}$, which is convergent to $v \in \mathscr{E}_{1}$, in the sense of distributions, and such that sequence $\left\{v_{j} d \mu\right\}$ is weakly 
convergent to some measure $v$. Then we have by inequality (6) that there exists a constant $A>0$ such that

$$
\int_{\Omega}\left(-v_{j}\right)^{2} d \mu \leq A e_{1}\left(v_{j}\right)^{\frac{2}{n+1}} \leq A .
$$

Thus, $v_{j} \in L^{2}(\mu)$. Therefore, there exists a finite convex combination of $v_{j}$, denote this by $w_{j}$, such that $\left\{w_{j}\right\} \subseteq \mathscr{E}_{1}$ converges to some function $w \in L^{2}(\mu)$. Furthermore, $d v=w d \mu$. But $\left\{v_{j}\right\}$ is weakly convergent to $\left(\lim \sup v_{j}\right)^{*}$ w.r.t. the Lebesgue measure, and therefore $\left\{w_{j}\right\}$ is weak convergent to $\left(\lim \sup w_{j}\right)^{*}=\left(\lim \sup v_{j}\right)^{*}$. Hence, $w=\left(\lim \sup v_{j}\right)^{*}$, where $(y)^{*}$ denotes the upper semicontinuous regularization of $y$.

The uniqueness part follows from Proposition 3.1, and therefore it remains to prove that there exists a minimizer of $\mathscr{J}_{\mu}$. Theorem B yields that exists a function $u \in \mathscr{E}_{1}$ such that $\left(d d^{c} u\right)^{n}=\mu$. Let $\left\{u_{j}\right\} \subseteq \mathscr{E}_{1}$ be such that $\lim _{j \rightarrow \infty} \mathscr{J}_{\mu}\left(u_{j}\right)=\inf _{w \in \mathscr{E}_{1}} \mathscr{J}_{\mu}(w)$. Using Lemma 2.2 together with what we just have proved, we can pick a subsequence again denoted by $\left\{u_{j}\right\}$, and a function $u \in \mathscr{E}_{1}$ such that

$$
\int_{\Omega}\left|u_{j}-u\right| d \mu \rightarrow 0 \quad \text { as } \quad j \rightarrow \infty .
$$

Set $v_{k}=\left(\sup _{j \geq k} u_{j}\right)^{*}$. Then it follows that $v_{k} \geq u_{k}$, which implies that $e_{1}\left(v_{k}\right) \leq e_{1}\left(u_{k}\right)$ (see, e.g., [10] or Lemma 6.1 in [4]). Thus, $v_{k} \in \mathscr{E}_{1}$. The decreasing sequence $\left\{v_{k}\right\}$ converges to $u$, as $j \rightarrow \infty$, and $e_{1}\left(v_{k}\right) \rightarrow e_{1}(u)$, as $k \rightarrow \infty$. The monotone convergence theorem implies that $\lim _{k \rightarrow \infty} \int_{\Omega} v_{k} d \mu=$ $\int_{\Omega} v d \mu$. Therefore, we have that

$$
e_{1}(u)=\lim _{j \rightarrow \infty} e_{1}\left(v_{j}\right)=\liminf _{j \rightarrow \infty} e_{1}\left(v_{j}\right) \leq \liminf _{j \rightarrow \infty} e_{1}\left(u_{j}\right) .
$$

Hence,

$$
\liminf _{j \rightarrow \infty} \mathscr{J}_{\mu}\left(u_{j}\right)=\liminf _{j \rightarrow \infty} e_{1}\left(u_{j}\right)+\lim _{j \rightarrow \infty} \int_{\Omega} u_{j} d \mu \geq e_{1}(u)-\|u\|_{1}=\mathscr{J}_{\mu}(u) .
$$

Thus, $u$ is a minimizer.

Proof of Theorem A. (3) $\Rightarrow$ (2) Assume that condition (2) is not satisfied. Then for all $j$ there exists a sequence $\left\{u_{j}\right\} \subseteq \mathscr{E}_{1}$ with

$$
\int_{\Omega}\left(-u_{j}\right) d \mu \geq j e_{1}\left(u_{j}\right)^{\frac{1}{1+n}} .
$$


Without loss of generality we can assume that $e_{1}\left(u_{j}\right)=1$. Let $v_{j}=\sum_{l=1}^{j} \frac{1}{l^{2}} u_{l}$, then by Lemma 2.2 we have that

$$
e_{1}\left(v_{j}\right)^{\frac{1}{n+1}} \leq \sum_{l=1}^{j} \frac{1}{l^{2}} e_{1}\left(u_{l}\right)^{\frac{1}{n+1}}=\sum_{l=1}^{j} \frac{1}{l^{2}},
$$

which implies that $v=\sum_{l=1}^{\infty} \frac{1}{l^{2}} u_{l} \in \mathscr{E}_{1}$. On the other hand,

$$
\int_{\Omega}(-v) d \mu \geq \sum_{l=1}^{\infty} \frac{1}{l^{2}} \int_{\Omega}\left(-u_{l}\right) d \mu \geq \sum_{l=1}^{\infty} \frac{l}{l^{2}}=\infty .
$$

Thus, $v \notin L^{1}(\mu)$, and a contradiction has been obtained.

(2) $\Rightarrow$ (1) In this part of the proof we follow the idea from Theorem 5.1 in [10]. Assume that $\mu$ is a non-negative Radon measure such that (1) holds. Assume first that $\mu$ is supported by a compact set $K \Subset \Omega$, and let $h_{K}$ denote the relative extremal function for $K$ (see, e.g., [19] for the definition of $h_{K}$ ). Set

$$
\mathscr{M}=\left\{v \geq 0: \operatorname{supp} v \subset K, \int_{\Omega}(-\varphi)^{2} d v \leq C e_{1}(\varphi)^{\frac{2}{n+1}} \text { for all } \varphi \in \mathscr{E}_{1}\right\},
$$

where $C>2 e_{1}\left(h_{K}\right)^{\frac{n-1}{n+1}}$ is a fixed constant. For a compact set $L \subset K$ we have that

$$
h_{K} \leq h_{L}, \quad \text { and } \quad e_{1}\left(h_{L}\right) \leq e_{1}\left(h_{K}\right) .
$$

Therefore, it follows that

$$
\begin{aligned}
\int_{\Omega}(-\varphi)^{2}\left(d d^{c} h_{L}\right)^{n} & \leq 2\left\|h_{L}\right\| \int_{\Omega}(-\varphi)\left(d d^{c} \varphi\right) \wedge\left(d d^{c} h_{L}\right)^{n-1} \\
& \leq 2\left(\int_{\Omega}(-\varphi)\left(d d^{c} \varphi\right)^{n}\right)^{\frac{2}{n+1}}\left(\int_{\Omega}\left(-h_{L}\right)\left(d d^{c} h_{L}\right)^{n}\right)^{\frac{n-1}{n+1}} \\
& \leq C e_{1}(\varphi)^{\frac{2}{n+1}} \quad \text { for all } \quad \varphi \in \mathscr{E}_{1} .
\end{aligned}
$$

Hence, for every compact set $L \subset K$ we have that $\left(d d^{c} h_{L}\right)^{n} \in \mathscr{M}$. Fix $v_{0} \in \mathscr{M}$ and define

$$
\begin{aligned}
& \mathscr{M}^{\prime}=\{v \geq 0: v(\Omega)=1, \operatorname{supp} v \subset K, \\
& \left.\quad \int_{\Omega}(-\varphi)^{2} d v \leq\left(\frac{C}{T}+\frac{C}{v_{0}(\Omega)}\right) e_{1}(\varphi)^{\frac{2}{n+1}} \text { for all } \varphi \in \mathscr{E}_{1}\right\},
\end{aligned}
$$


where $T=\sup \{v(\Omega): v \in \mathscr{M}\}$. Then we have for $v \in \mathcal{M}$ that

$$
\begin{aligned}
\int_{\Omega}( & \varphi)^{2} \frac{\left.(T-v(\Omega)) d v_{0}+v_{0}(\Omega) d v\right)}{T v_{0}(\Omega)} \\
& \leq \int_{\Omega}(-\varphi)^{2} d v_{0} \frac{T-v(\Omega)}{T \nu_{0}(\Omega)}+\frac{1}{T} \int_{\Omega}(-\varphi)^{2} d v \\
& \leq\left(C \frac{T-v(\Omega)}{T v_{0}(\Omega)}+\frac{C}{T}\right) e_{1}(\varphi)^{\frac{2}{n+1}} \\
& \leq\left(\frac{C}{v_{0}(\Omega)}+\frac{C}{T}\right) e_{1}(\varphi)^{\frac{2}{n+1}} \quad \text { for all } \quad \varphi \in \mathscr{E}_{1} .
\end{aligned}
$$

Hence,

$$
\frac{\left.(T-v(\Omega)) v_{0}+v_{0}(\Omega) \nu\right)}{T v_{0}(\Omega)} \in \mathscr{M}^{\prime} \quad \text { for all } \quad v \in \mathscr{M}
$$

Thus, $\mathscr{M}^{\prime}$ is a convex and weak ${ }^{*}$-compact set of probability measures. Then it follows from [22] that there exists a function $f \in L^{1}(\mu)$, and a measure $v \in \mathscr{M}^{\prime}$ such that $\mu=f d v+v_{s}$, where $v_{s}$ is orthogonal to $\mathscr{M}^{\prime}$. Note that since $\left(d d^{c} h_{L}\right)^{n} \in \mathscr{M}$, then all measures orthogonal to $\mathscr{M}^{\prime}$ must be carried by a pluripolar sets. Thus, $v$ identically 0 , since $\mu$ vanishes on pluripolar sets. Furthermore, by Lemma 4.1, we know that for each $\mu \in \mathscr{M}^{\prime}$ there exists a uniquely determined function $u \in \mathscr{E}_{1}$ with $\left(d d^{c} u\right)^{n}=\mu$.

For each $j$ let $\mu_{j}$ be a non-negative Radon measure defined by $\mu_{j}=$ $\min (f, j) d v$. The measure $\mu_{j}$ satisfy inequality (6), since $v$ have this property. Therefore there exists a unique function $u_{j} \in \mathscr{E}_{1}$ with $\left(d d^{c} u_{j}\right)^{n}=d \mu_{j}$. Theorem 4.5 in [10] yields that $\left\{u_{j}\right\}$ is a decreasing sequence that converges pointwise to a function $u \in \mathscr{E}_{1}$ with $\left(d d^{c} u\right)^{n}=\mu$. Finally, if $\mu$ only satisfy (1), let $\left\{K_{j}\right\}$ be an increasing sequence of compact subsets of $\Omega$ with $\Omega=\bigcup_{j} K_{j}$, and set $\mu_{j}=\chi_{K_{j}} d \mu$. We can then proceed as before.

(1) $\Rightarrow$ (4) Assume that there exists a function $u \in \mathscr{E}_{1}$ such that $\left(d d^{c} u\right)^{n}=\mu$. Let $\left\{v_{j}^{\prime}\right\} \subseteq \mathscr{E}_{1}$ be a sequence with $e_{1}\left(v_{j}^{\prime}\right) \leq 1,\left\{v_{j}^{\prime}\right\}$ converges in the sense of distributions, and select a weak*-convergent subsequence $\left\{v_{j} d \mu\right\}$ of $\left\{v_{j}^{\prime} d \mu\right\}$ converging to $d v$ for some measure $v \leq 0$. Let $\psi \in C_{0}^{\infty}(\Omega), 0 \leq \psi \leq 1$. Then we have that $\psi\left(d d^{c} u\right)^{n} \leq\left(d d^{c} u\right)^{n}$. Then we have that the measure $\psi\left(d d^{c} u\right)^{n}$ satisfies condition (2), and therefore also (1). Thus, there exists a function $\varphi \in \mathscr{E}_{1}$ such that $\left(d d^{c} \varphi\right)^{n}=\psi\left(d d^{c} u\right)^{n}$, and from the proof of the implication 
(2) $\Rightarrow(1)$ it follows that $u \leq \varphi$. This, together with Theorem 2.1 yields that

$$
\begin{aligned}
\int_{\Omega}\left(-v_{j}\right) \psi d \mu & =\int_{\Omega}\left(-v_{j}\right)\left(d d^{c} \varphi\right)^{n} \leq e_{1}\left(v_{j}\right)^{\frac{1}{n+1}}\left(\int_{\Omega}(-\varphi)\left(d d^{c} \varphi\right)^{n}\right)^{\frac{n}{n+1}} \\
& \leq e_{1}\left(v_{j}\right)^{\frac{1}{n+1}}\left(\int_{\Omega}(-u) \psi\left(d d^{c} u\right)^{n}\right)^{\frac{n}{n+1}} \\
& =e_{1}\left(v_{j}\right)^{\frac{1}{n+1}}\left(\int_{\Omega}(-u) \psi d \mu\right)^{\frac{n}{n+1}} .
\end{aligned}
$$

This means that $d \nu$ is absolutely continuous w.r.t. $d \mu$, and therefore there exists a function $f \in L^{1}(\mu), f \leq 0$, such that $d \nu=f d \mu$. Now let $\psi$ be a function defined on $\Omega$ with $0 \leq \psi \leq 1$. By a similar argument as above, we get that

$$
\int_{\Omega} \psi f d \mu=\lim _{j \rightarrow \infty} \int_{\Omega} \psi v_{j} d \mu
$$

(Choose $\psi_{\varepsilon} \in C_{0}^{\infty}(\Omega)$ so that $\int\left|\psi-\psi_{\varepsilon}\right|(-f-u) d \mu \leq \varepsilon$ and continue as above.)

Hence, there exist finite convex combinations of $v_{j}$, denoted by $w_{j}$, that converges to $f$ in $L^{1}(\mu)$. Therefore there exists a subsequence $\left\{w_{j_{k}}\right\}$ of $\left\{w_{j}\right\}$ that converges to $f$ a.e. w.r.t. $[\mu]$. From now on we shall use the notation $\left\{w_{j}\right\}$ instead of $\left\{w_{j_{k}}\right\}$. Set $v=\lim _{j \rightarrow \infty}\left(\sup _{k \geq j} v_{k}\right)^{*}$, then it follows from Fatou's lemma that $f \leq v$. Furthermore, we get that $\lim _{j \rightarrow \infty}\left(\sup _{k \geq j} w_{k}\right)^{*}=v$, since $\left\{v_{j}^{\prime}\right\},\left\{v_{j}\right\}$ and $\left\{w_{j}\right\}$ converge as distributions to the same limit $v \in \mathscr{E}_{1}$, and

$$
\int_{\Omega} v d \mu=\int_{\Omega} \lim _{j \rightarrow \infty}\left(\sup _{k \geq j} w_{k}\right)^{*} d \mu=\int_{\Omega} \lim _{j \rightarrow \infty}\left(\sup _{k \geq j} w_{k}\right) d \mu=\int_{\Omega} f d \mu .
$$

Thus, $v=f=\lim _{j \rightarrow \infty}\left(\sup _{k \geq j} v_{k}^{\prime}\right)$ a.e. w.r.t. [ $\mu$ ], and it follows from (7) that

$$
\lim _{j \rightarrow \infty} \int_{\Omega} v_{j} d \mu=\int_{\Omega} v d \mu
$$

Then

$$
\int_{\Omega}\left|v_{j}-v\right| d \mu \leq \int_{\Omega}\left(\max _{k \geq j} v_{k}-v_{j}\right) d \mu+\int_{\Omega}\left(\max _{k \geq j} v_{k}-v\right) d \mu=I_{1}+I_{2} .
$$

But $I_{1}$ converges to 0 , as $j \rightarrow \infty$, since

$$
\lim _{j \rightarrow \infty} \int_{\Omega} \max _{k \geq j} v_{k} d \mu=\lim _{j \rightarrow \infty} \int_{\Omega} v_{j} d \mu,
$$


and $I_{2}$ converges to 0 by the monotone convergence theorem. Thus,

$$
\lim _{j \rightarrow \infty} \int_{\Omega}\left|v_{j}-v\right| d \mu=0,
$$

i.e. $v_{j}$ converges to $v$ in $L^{1}(\mu)$. In other words, every subsequence of $\left\{v_{j}^{\prime}\right\}$ contains a subsequence that converges to $v=f=\lim _{j \rightarrow \infty}\left(\sup _{k \geq j} v_{k}^{\prime}\right)$ in $L^{1}(\mu)$.

\section{REFERENCES}

1. Åhag, P., Cegrell, U., Czyż, R., and Pham, H. H., Monge-Ampère measures on pluripolar sets, J. Math. Pures Appl. (9) 92 (2009), 613-627.

2. Åhag, P., Cegrell, U., Kołodziej, S., Pham, H. H., and Zeriahi, A., Partial pluricomplex energy and integrability exponents of plurisubharmonic functions, Adv. Math. 222 (2009), 2036-2058.

3. Åhag, P., and Czyż, R., An inequality for the beta function with application to pluripotential theory, J. Inequal. Appl. 2009, Art. ID 901397, 8 pp.

4. Åhag, P., and Czyż, R., Modulability and duality of certain cones in pluripotential theory, J. Math. Anal. Appl. 361 (2010), 302-321.

5. Åhag, P., Czyż, R., and Phạm, H. H., Concerning the energy class $\mathscr{E}_{p}$ for $0<p<1$, Ann. Polon. Math. 91 (2007), 119-130.

6. Bedford, E., and Taylor, B. A., Variational properties of the complex Monge-Ampère equation I. Dirichlet principle, Duke Math. J. 45 (1978), 375-403.

7. Bedford, E., and Taylor, B. A., Variational properties of the complex Monge-Ampère equation II. Intrinsic norms, Amer. J. Math. 101 (1979), 1131-1166.

8. Bedford, E., and Taylor, B. A., A new capacity for plurisubharmonic functions, Acta Math. 149 (1982), 1-40.

9. Berman, R. J., Boucksom, S., Guedj, V., and Zeriahi, A., A variational approach to complex Monge-Ampère equations, arXiv:0907.4490.

10. Cegrell, U., Pluricomplex energy, Acta Math. 180 (1998), 187-217.

11. Cegrell, U., The general definition of the complex Monge-Ampère operator, Ann. Inst. Fourier (Grenoble) 54 (2004), 159-179.

12. Cegrell, U., Approximation of plurisubharmonic function in hyperconvex domains, pp. 125129 in: Complex Analysis and Digital Geometry, Proc. Uppsala 2006, Acta Universitatis Upsaliensis 86, Uppsala 2009.

13. Cegrell, U., A general Dirichlet problem for the complex Monge-Ampère operator, Ann. Polon. Math. 94 (2008), 131-147.

14. Cegrell, U., Kołodziej, S., and Zeriahi, A., Subextension of plurisubharmonic functions with weak singularites, Math. Z. 250 (2005), 7-22.

15. Cegrell, U., and Persson, L., An energy estimate for the complex Monge-Ampère operator, Ann. Polon. Math. 67 (1997), 95-102.

16. Czyż, R., The complex Monge-Ampère operator in the Cegrell classes, Dissertationes Math. 466 (2009), 83 pp.

17. Diller, J., Dujardin, R., and Guedj, V., Dynamics of meromorphic maps with small topological degree II: Energy and invariant measure, Comment. Math. Helv. 86 (2011), 277-316.

18. Kalina, J., Some remarks on variational properties of inhomogeneous complex Monge-Ampère equation, Bull. Polish Acad. Sci. Math. 31 (1983), 9-13. 
19. Klimek, M., Pluripotential Theory, London Math. Soc. Monogr. 6, Oxford Univ. Press, New York 1991.

20. Kołodziej, S., The complex Monge-Ampère equation and pluripotential theory, Mem. Amer. Math. Soc. 178 (2005), no. 840.

21. Persson, L., A Dirichlet principle for the complex Monge-Ampère operator, Ark. Mat. 37 (1999), 345-356.

22. Rainwater, J., A note on the preceding paper, Duke Math. J. 36 (1969), 799-800.

DEPARTMENT OF MATHEMATICS

AND MATHEMATICAL STATISTICS

UMEÅ UNIVERSITY

SE-901 87 UMEÅ

SWEDEN

E-mail: Per.Ahag@math.umu.se

Urban.Cegrell@math.umu.se
INSTITUTE OF MATHEMATICS

JAGIELLONIAN UNIVERSITY

ŁOJASIEWICZA 6

30-348 KRAKÓW

POLAND

E-mail: Rafal.Czyz@im.uj.edu.pl 